\title{
Union européenne et science politique : où en est le débat théorique?
}

\section{Christian Lequesne et Andy SMITH}

\section{(2) OpenEdition \\ 1 Journals}

\section{Édition électronique}

URL : http://journals.openedition.org/conflits/377

DOI : $10.4000 /$ conflits.377

ISSN : $1777-5345$

Éditeur :

CCLS - Centre d'études sur les conflits lilberté et sécurité, L'Harmattan

\section{Édition imprimée}

Date de publication : 15 janvier 1997

ISSN : 1157-996X

Référence électronique

Christian Lequesne et Andy SMITH, «Union européenne et science politique : où en est le débat théorique? », Cultures \& Conflits [En ligne], 28 | hiver 1997, mis en ligne le 16 mars 2006, consulté le 30 mars 2021. URL : http://journals.openedition.org/conflits/377 ; DOI : https://doi.org/10.4000/conflits. 377

Ce document a été généré automatiquement le 30 mars 2021.

Creative Commons License 


\title{
Union européenne et science politique : où en est le débat théorique?
}

\author{
Christian Lequesne et Andy SMITH
}

\section{Christian LEQUESNE, Andy SMITH ${ }^{12}$}

Dans un article de presse récent ${ }^{3}$, Gilles Tordjman fustigeait " le silence de la science politique " sur la situation socio-économique de la France: " Et si 'la pensée unique', ennemi commode et consensuel, n'était pas le néo-libéralisme terne et tempéré, mais bien la vieille analyse politique crispée sur des modus operandi que plus personne ne remet en cause? ". Pour un politiste, une première réaction naturelle consisterait à renvoyer cet " étranger au sérail ", à la lecture d'une quantité d'ouvrages récents qui sortent des sentiers battus. En y pensant de plus près, la critique du journaliste mérite néanmoins d'être considérée sans dédain. La science politique n'a-t-elle pas pour vocation de comprendre les racines des enjeux sociaux et non de relater la seule description détaillée de leur interprétation par les acteurs? Sans que l'attaque de Tordjman vise particulièrement l'analyse de l'intégration européenne, elle résume une interrogation essentielle de la recherche sur cet objet : comment rendre compte du fait que l'intégration européenne n'est pas seulement un défi pour le monde de l'action mais aussi un défi pour l'intelligibilité de ce monde? Chacun à leur manière, les chapitres de ce numéro spécial abordent ce défi. Par là même, ils démontrent la fécondité d'une confrontation d'approches entre des chercheurs qui définissent, pour l'essentiel d'entre eux, leurs travaux soit à partir des relations internationales, soit à partir de l'étude des politiques publiques. En les lisant, on se rend vite compte que l'intégration européenne - comme objet de recherche et certainement aussi comme objet politique - déstabilise les découpages hérités du passé et que ni les relations internationales ni les politiques publiques n'échappent à ce mouvement ${ }^{4}$. Mettre en relief les principales approches théoriques actuellement utilisées par les analystes de l'intégration européenne constitue l'ambition principale de cet article introductif rédigé par deux chercheurs qui non seulement mènent des recherches empiriques sur 
l'intégration européenne, mais sont aussi amenés à l'enseigner, se trouvant dès lors confrontés au fait que la littérature théorique existante n'aide pas toujours à éclaircir leurs observations empiriques. Dans cette perspective, et contrairement à ce que disent encore certains manuels de science politique au chapitre " intégration européenne ", l'heure n'est plus à une opposition entre une école intergouvernementaliste et une école néofonctionnaliste issues des relations internationales. A mesure que les travaux sur l'intégration européenne s'accumulent, les clivages se sont complexifiés et réduisent les découpages trop stricts au sein et entre les sous-disciplines de la science politique. Il n'en demeure pas moins que deux approches génériques continuent de dominer l'étude de l'intégration européenne. La première met l'accent sur l'action entre des Etats-Nations, l'autre sur l'émergence d'un espace d'action politique dont les composants se situent au-delà des seuls Etats. Ne voulant pas céder à la tentation commode de l'éclectisme, notre propos n'est pas de dégager une " troisième voie " entre ces deux écoles. Sans aucun doute plus proches par nos travaux actuels de la seconde que de la première, notre objectif est de les critiquer toutes les deux afin de poser ensuite, dans un article de conclusion, quelques jalons d'une approche qui ne découlerait plus directement des apports et des limites de l'une et de l'autre. L'Europe de l'" intergouvernementalisme libéral " : des limites du stato-centrisme A l'instar des pièces de music-hall, les théories des relations internationales appliquées à l'intégration européenne (genre originellement américain) ont connu depuis quarante ans des " up " et des " down ", des " out " et des " come back " fortement liés aux cycles historiques de la construction européenne. Après avoir été le paradigme dominant sur la scène scientifique américaine pendant une quinzaine d'années, le néofonctionnalisme visant à expliquer le développement de l'intégration européenne à partir d'une convergence des élites a ainsi connu un incontestable déclin au début des années soixante-dix. Ce déclin correspond à la prise en compte par bon nombre de politistes d'un retour de l'Etat matérialisé par la " crise de la chaise vide ", par la montée en puissance du Conseil européen ou encore par la politique d'obstruction du gouvernement britannique à l'égard du budget communautaire. Principal chef de file des néofonctionnalistes américains, Ernst Haas, publia ainsi au milieu des années soixante-dix un ouvrage autocritique qu'il intitula fort explicitement The obsolescence of regional integration theory ${ }^{5}$. Retraçant plus récemment les évolutions du débat théorique sur l'Union européenne au sein de la science politique, deux auteurs américains n'ont pas hésité non plus à qualifier la période allant du début des années soixante-dix au milieu des années quatre-vingt " d'âge sombre du développement des théories de l'intégration ". Ils ajoutaient cependant qu'un regain pour la " grand theory " avait accompagné l'adoption en 1985 du " Livre Blanc sur le marché intérieur " 6 . Le retour de la " grand theory " Une forme de " régénération " théorique a effectivement correspondu, dans le contexte universitaire américain mais aussi européen, à la signature en 1986 de l'Acte Unique Européen. Ce mouvement s'est toutefois manifesté dans un environnement marqué par un déplacement de paradigmes qui avaient jusque là emprunté quasi-exclusivement à la théorie des relations internationales vers la comparative politics ${ }^{7}$, voire le judicial politics ${ }^{8}$. Dans son article de West European Politics publié en 1994, Simon Hix en souligne fort justement les raisons : les théories des relations internationales permettent d'analyser les conflits politiques au sein de la Communauté européenne uniquement à partir de la matrice linéaire soutien ou opposition à l'intégration supranationale, alors que ces conflits portent de plus en plus sur des " questions d'allocation et de distribution des ressources " que la comparative 
politics est mieux à même d'interpréter. Alors que les années quatre-vingt se caractérisent par la forte séduction des théoriciens américains des relations internationales pour les phénomènes transnationaux 9 , l'approche intergouvernementaliste - qu'avait développée Stanley Hoffmann dès les années soixante en réaction au néofonctionnalisme $\mathrm{e}^{10}$ - se maintient dans le domaine de l'Union européenne en étant souvent intégrée à des problématiques plus éclectiques. Toute une série de travaux l'illustrent : ceux de Stanley Hoffmann et de Bob Keohane inspirés de la " théorie des régimes "11, certains travaux de Geoffrey Garrett inspirés des choix rationnels ${ }^{12}$ et surtout ceux d'Andrew Moravcsik qualifiés par l'auteur lui-même d'" intergouvernementalisme libéral ". Unité et rationalité de l'acteur étatique Nous faisons le choix d'une lecture critique des seuls travaux de A. Moravcsik car ce sont ceux qui vont le plus loin dans la prétention de formuler une théorie générale de l'intégration européenne. Rejetant toute démarche qui consisterait à penser la recherche scientifique uniquement " en réaction à ", nous ne visons surtout pas à affirmer que l'intergouvernementalisme libéral ne présente aucun intérêt parce qu'il valorise un acteur étatique qui ne compte plus dans le processus d'intégration européenne ${ }^{13}$. Notre critique porte plutôt sur les limites d'une entreprise théorique qui, à cause d'une excessive rationalisation, est trop monocausale et dès lors ne reflète que partiellement l'originalité du politique au sein de l'Union européenne ${ }^{14}$. C'est dans un article du Journal of Common Market Studies, paru en 1993, qu'A. Moravcsik a donné la définition la plus précise de ce qu'il a appelé " une approche intergouvernementaliste libérale " de l'Union européenne ${ }^{15}$. Son approche repose principalement sur trois hypothèses qui ont le statut de postulat épistémologique : la rationalité de l'acteur étatique ; l'exercice du pouvoir politique comme résultante d'une négociation entre les gouvernements des Etats ; une théorie dite " libérale " de la formation des préférences sociétales. La démonstration consiste alors à tester ces hypothèses sur la réalité empirique en utilisant un raisonnement hypothético-déductif. S'inscrivant dans l'héritage du néoréalisme classique, les deux premières hypothèses avaient déjà été exploitées par A. Moravcsik dans un article d'International Organization consacré en 1991 à la genèse de l'Acte Unique Européen ${ }^{16}$. L'auteur y décrivait une Communauté européenne au sein de laquelle le pouvoir politique résultait de la confrontation d'intérêts nationaux entre des acteurs étatiques appelés la France, l'Allemagne, le Royaume-Uni... Dans un contexte d'interdépendance croissante de l'économique et du politique, ces Etats n'avaient guère d'autre choix que la mise en commun de leurs intérêts nationaux en vue de satisfaire la sécurité et le bien-être de leurs citoyens. Pour A. Moravcsik comme pour la plupart des intergouvernementalistes, Etat et gouvernement central sont ainsi très largement confondus. D'où une première critique pouvant lui être adressée : peut-on encore parler de l'Etat sans le considérer comme un ensemble pluriel de segments et de jeux de pouvoir bureaucratiques ou encore comme un système multi-organisationnel ? En " disséquant " l'Etat dans le contexte de l'Union européenne, on constate en effet assez vite que les acteurs qui le composent reflètent des intérêts et des logiques de pouvoir qui sont loin d'être toujours agrégées ou convergentes. Bien que séduit par l'intergouvernementalisme libéral, Elie Cohen a ainsi démontré dans un récent ouvrage qu'on ne pouvait pas comprendre les positions de l'Etat français à l'égard de la déréglementation européenne des télécommunications sans analyser finement les redéploiements qu'ont induit les normes de droit et de marché pour les différents corps composant l'appareil politico-administratif ${ }^{17}$. Il a observé en particulier une " montée d'experts juridiques souvent issus du Conseil 
d'Etat, là où régnaient en maîtres les ingénieurs des corps techniques ou les administrateurs des grands corps des finances "18. De toute évidence, le positionnement de l'Etat français face à l'Europe des télécommunications ne peut pas être analysé sans une étude de la redéfinition des hiérarchies de pouvoir qu'induit ce projet pour les différents acteurs le composant. La confusion Etat-gouvernement central provient en second lieu du fait qu'A. Moravcsik aborde l'Union européenne essentiellement à partir des " grand bargains " que sont le traité de Rome, le Système monétaire européen, l'Acte Unique Européen ${ }^{19}$. S'il s'intéressait aux politiques communautaires avec un champ d'analyse plus micro (et plus particulièrement encore à leur mise en œuvre), il verrait poindre plus clairement une diversité interne de l'Etat qui contredirait bien vite les hypothèses de l'unité et de la rationalité. A. Moravcsik est conduit en troisième lieu à considérer les politiques produites par l'Union européenne essentiellement comme le résultat d'inter-state bargainings reposant sur un " plus petit commun dénominateur ". Voilà un autre point critiquable de son analyse qui résulte d'une insuffisante prise en compte de l'évolution des modes opératoires de la décision depuis que l'Acte Unique Européen, puis les traités de Maastricht et d'Amsterdam, ont réhabilité le vote majoritaire dans un nombre important de domaines : marché intérieur, recherche et technologie, politique sociale... Ce manque s'explique par le fait qu'en souscrivant au postulat d'un Etat unitaire et souverain, A. Moravcsik est finalement amené à valoriser le principe unanimitaire qui, certes, régit encore dans des domaines essentiels comme la politique étrangère et la sécurité commune, la révision constitutionnelle des traités ou l'harmonisation des fiscalités. Il renoue alors implicitement avec cette distinction aléatoire entre high politics et low politics que Stanley Hoffmann avait opérée en son temps. L'hypothèse $d u$ " plus petit commun dénominateur " conduit en outre A. Moravcsik à conférer une importance déterminante aux " grands " Etats sur les " petits " Etats dans la négociation communautaire. Analysant la genèse de l'Acte Unique Européen, il écrit ainsi : " le résultat représente la convergence des choix de politique intérieure des grands Etats membres "20, à savoir l'Allemagne du Chancelier Kohl (supporter de tout développement de la Communauté européenne), la France du Président Mitterrand (suite à la " conversion " en 1983 des socialistes à l'économie du marché) et la Grande-Bretagne du Premier ministre Thatcher (attirée par tout projet visant à libéraliser l'économie). Cette valorisation des " grands " opérée par A. Moravcsik néglige alors le jeu des alliances mis à jour par une série de travaux sur la théorie et la pratique de la négociation intergouvernementale au sein de l'Union européenne $^{21}$. Il ressort en effet assez clairement de ces travaux que la principale caractéristique du processus multilatéral de négociation est d'offrir à l'ensemble des acteurs gouvernementaux la possibilité de peser sur la distribution des gains au-delà de leurs inégalités de ressources et de statuts. Les débats houleux entre représentants des " grands " et des " petits " Etats sur la réforme de la pondération des voix au Conseil, qui ont marqué la révision du traité de Maastricht, en sont une illustration récente. En limitant l'exercice du pouvoir au sein de l'Union européenne à des inter-state bargainings dans lesquels s'imposent surtout les " grands " Etats, Moravcsik est conduit en outre à sous-estimer l'autonomie des autres acteurs que sont les institutions communautaires et les acteurs sociaux organisés transnationalement. Nous souscrivons bien volontiers à sa critique selon laquelle les approches de l'Union européenne en termes de gouvernance à niveaux multiples (multilevel governance) pèchent souvent par un excès de pluralisme qui les amènent à confondre l'activité politique déployée par des réseaux multiples d'acteurs (Etats, gouvernements subnationaux, institutions 
communautaires, acteurs sociaux) et l'exercice du pouvoir politique (cf. infra) ${ }^{22}$. L'analyse du pouvoir au sein de l'Union européenne nécessite en effet de dégager les formes d'autorité exercées par certains acteurs sur d'autres et donc de mener une analyse fine des hiérarchies qui se construisent dans la négociation continue. Rien n'autorise néanmoins à considérer, comme le fait Moravcsik, que seuls les Etats se trouvent au sommet de ces hiérarchies. Les institutions communautaires (la Commission, le Parlement européen et la Cour de justice) n'ont a contrario pas seulement une fonction instrumentale visant à " accroître le pouvoir des gouvernements en élargissant d'une part l'efficacité des marchandages interétatiques et [...] d'autre part l'autonomie des responsables politiques vis-à-vis des groupes particuliers qui composent l'arène politique nationale "23. Elles ont aussi une capacité à générer des intérêts et des valeurs propres pouvant dépasser et défier les Etats qui les ont créées ${ }^{24}$. Seule une opération de déconstruction des institutions communautaires à laquelle A. Moravcsik ne procède jamais- permet cependant de saisir leur constitution progressive en acteurs complexes. Pour ce qui est par exemple de la Commission, il s'agit dès lors moins d'étudier son action que celle des commissaires, des cabinets et des directions générales constituant ce que L. Cram a appelé une " multi-organisation "25. Une cinquième critique porte sur la dimension dite libérale $\mathrm{du}$ " liberal intergovernmentalism " qui distingue a priori A. Moravcsik des théoriciens néoréalistes des relations internationales. Si ce dernier conçoit en effet les inter-state bargainings comme une confrontation d'intérêts nationaux, il y voit néanmoins le résultat de demandes sociétales agrégées à l'échelon de chaque Etat. A l'instar d'autres auteurs ${ }^{26}$, A. Moravcsik est ainsi légitimement convaincu qu'on ne saurait étudier l'Union européenne sans se livrer à une analyse minutieuse de la politique intérieure des différents Etats qui la composent. Cette prise en compte du rapport interne-externe le conduit à élaborer une théorie de la marge de manœuvre des gouvernements, pris entre les contraintes sociales internes d'une part et les contraintes de la coopération européenne d'autre part, qui n'est pas sans évoquer le " two level games " de Robert Putnam ${ }^{27}$. Au final, l'Union européenne accroîtrait le pouvoir des Etats en permettant aux dirigeants gouvernementaux d'utiliser la contrainte de la coopération internationale pour contourner certaines demandes formulées au plan interne par les parlements, les groupes d'intérêts, etc. ${ }^{28}$. L'hypothèse est intéressante car les dirigeants politico-administratifs nationaux invoquent effectivement de façon régulière la contrainte européenne pour imposer aux acteurs sociaux des mesures contestées au plan interne ${ }^{29}$, voire pour briser la résistance au changement de certains groupes réputés trop puissants (comme les syndicats de la métallurgie dans les années soixante-dix ou ceux de l'aviation commerciale plus récemment). Postuler néanmoins que l'Etat demeure la seule scène d'agrégation des demandes sociales à partir d'un schéma où des " donneurs d'ordre sociétaux délèguent leur pouvoir (ou d'une autre manière les contraignent) à des agents gouvernementaux " est très insuffisant ${ }^{30}$. Ce schéma néglige en effet le mouvement de transnationalisation des acteurs sociaux qui, de plus en plus, projettent leurs intérêts dans l'espace politique extra-étatique sans avoir obligatoirement recours à la médiation des Etats. Bien entendu, l'émergence d'une scène européenne de représentation des intérêts sociaux ne signifie pas la disparition des scènes corporatistes nationales mais plutôt un dédoublement ${ }^{31}$ qui est délibérément ignoré par Moravcsik. Lorsqu'il écrit que " le programme marché intérieur, à l'instar de la Communauté européenne trente ans auparavant, semble avoir été lancé indépendamment de toute pression des groupes d'intérêt transnationaux " ${ }^{132}$, 
il exclut ainsi de manière trop catégorique l'impact qu'ont pu avoir des organisations comme l'" Union Nationale des Industries de la Communauté Européenne " (UNICE) ou l'" European Round Table " sur l'inscription de cette politique à l'agenda de la Communauté $^{33}$. En outre, la conception qu'a A. Moravcsik des préférences nationales est essentiellement positiviste. Par conséquent, elle ne tient absolument pas compte des dimensions, idéologique et historique, qui sont attachées à tout processus de formation d'une demande sociale ou d'un intérêt ${ }^{34}$. Une réification du politique Face à l'Union européenne, l'intergouvernementalisme libéral souffre enfin d'un handicap essentiel : il procède d'une conception réifiée du pouvoir politique qui amène à postuler comme des données initiales de l'intégration européenne des notions directement issues des théories politiques de l'Etat-Nation comme la souveraineté ou encore la soumission hiérarchique de " principals " à des " agents ". A l'inverse de cela, tout l'effort du chercheur nous semble devoir consister à s'extraire de ces canons normatifs pour re-questionner complètement le " comment " de l'exercice du pouvoir. A cet égard, les travaux de A. Moravcsik résument assez bien la gamme classique des questions que se posent généralement les political scientists américains face à un objet de recherche. Comme l'a justement observé Pascal Vennesson ${ }^{35}$, il s'agit toujours d'explorer la causalité, d'exiger une définition précise des variables dépendantes et indépendantes, de mesurer la capacité prédictive d'une théorie (Moravcsik parle ainsi de " l'échec du néofonctionnalisme qui n'a pas su prédire l'évolution de la Communauté européenne " ${ }^{136}$ ) et surtout de valoriser la parcimonie dans l'utilisation des variables empiriques. Ce dernier postulat de recherche selon lequel la richesse phénoménale nuirait à la rigueur théorique ${ }^{37}$ conduit tout particulièrement à souligner les limites attachées à l'intergouvernementalisme libéral. En imposant un choix trop strict de " what matters? ", il empêche en effet de saisir la diversité des configurations d'acteurs et la dynamique différenciée des échanges politiques, des réseaux et des hiérarchies qui caractérisent l'Union européenne. L'Europe comme espace d'action politique: un deuxième souffle suffira-t-il? Depuis cinq à dix ans, l'approche intergouvernementaliste de l'intégration européenne, même sous sa forme dite " libérale ", se trouve mise en cause par un nombre croissant d'ouvrages et d'articles en Europe et, dans une moindre mesure, aux Etats-Unis. A l'inverse de l'accent mis sur les Etats " gardiens du temple ", ceux qui privilégient " l'européanisation " ou " l'européification " du politique en Europe ${ }^{38}$ soulignent qu'il est plus pertinent de se pencher sur les contours de ce que Pierre Muller a baptisé un " espace européen d'action publique "39. En formalisant un schéma sous-jacent qui est celui de " l'interdépendance ", cette approche a donné lieu à une quantité impressionnante d'études sur l'Union européenne " en action " partant des politiques publiques communautaires. Si le bilan empirique de ces études est largement positif, la modestie de certains aperçus théoriques laisse toutefois dans l'ombre plusieurs questions fondamentales. A ce propos, il est intéressant de noter que quelques spécialistes français de politiques publiques s'interrogent publiquement sur l'état de leur sousdiscipline en faisant appel à la quête d'un " deuxième souffle "40. Pour ce qui concerne la recherche sur l'Union européenne, nous ne sommes pas complètement convaincus que les mesures préconisées par ces auteurs critiques suffisent à déboucher sur un plus grand éclairage théorique de notre objet. La formalisation de l'interdépendance Développée à partir des années soixante-dix et quatre-vingt, l'analyse de l'intégration européenne prenant en compte la notion d'interdépendance souligne l'impact des processus de recomposition transfrontalière sur les modes d'action des acteurs 
économiques, politiques et sociaux ${ }^{41}$. Profondément empirique, l'hétérogénéité de son application découle en grande partie de trois sources d'inspiration différentes: la science administrative, la théorie des régimes et l'économie politique. Intéressés par l'émergence de nouvelles institutions et règles de droit, les spécialistes de science administrative ont progressivement ouvert leur recherche au processus de formulation ${ }^{42}$ et de mise en œuvre ${ }^{43}$ des politiques communautaires. Développée par Wolfgang Wessels, la théorie de " la fusion " des administrations nationales et européenne au travers de leurs interactions fonctionnelles est l'un des exemples les plus illustratifs de cette évolution ${ }^{44}$. La comparaison établie par Fritz Scharpf ${ }^{45}$ et plus récemment par Maurice Croisat et Jean-Louis Quermonne ${ }^{46}$ entre le système politique communautaire et les systèmes politiques nationaux relevant du "fédéralisme coopératif " constitue une autre piste de recherche représentative de ce courant d'analyse. Considérée à partir des années 1980 comme une théorie générale des relations internationales ${ }^{47}$, l'approche par les " régimes " constitue une deuxième source du schéma d'analyse interdépendantiste. Se demandant pourquoi les Etats adoptent des stratégies de coopération et d'intégration en l'absence de sanctions qui leur seraient directement applicables, elle met en doute l'indivisibilité de la souveraineté nationale. Formalisée à partir de la notion de " pooled sovereignty " ${ }^{48}$, la théorie des régimes envisage alors la Communauté européenne comme une série d'institutions sectorielles dans lesquelles se déroulent des jeux à somme positive. L'analyse interdépendantiste de l'intégration européenne se nourrit enfin de l'économie politique. Focalisés pendant quinze ans sur l'analyse conjoncturelle de la crise économique, l'hypothèse d'un déplacement structurel des économies occidentales a commencé en effet à interroger sérieusement les économistes de l'intégration communautaire au milieu des années quatre-vingt. Une conceptualisation de " la globalisation " des échanges et de la " menace concurrentielle " que représentent les entreprises situées dans d'autres régions géographiques que l'Europe (en particulier l'Asie) a débouché sur une réaffirmation des rapports entre capitalisme européen et construction politique. Pour Margaret Sharpe, les stratégies industrielles d'alliances et de coopérations transfrontalières au sein du projet dérégulateur du marché intérieur ont ainsi constitué les éléments moteurs de l'intégration communautaire au milieu des années quatre-vingt ${ }^{49}$. Le retournement de politique économique opéré en France par les socialistes à partir de 1983 est également cité souvent comme une preuve de la reconnaissance par les acteurs politiques nationaux de l'émergence d'une économie européenne interdépendante ${ }^{50}$. Trois idées rassemblent au fond ces approches disciplinaires différentes de l'intégration européenne (science administrative, théorie des régimes et économie politique) sous la bannière analytique de l'interdépendance : une conception transnationale des relations internationales; le primat accordé aux processus d'échange et de négociation politiques ${ }^{51}$; une vision de la dynamique de l'intégration communautaire conçue comme une série d'adaptations incrémentales plus que de ruptures. Depuis l'Acte Unique Européen, les approches interdépendantistes ont engendré, souvent indirectement, une quantité de recherches empiriques et ont ainsi contribué à accroître la connaissance du " comment " s'opère la régulation des multiples sphères d'action publique au sein de l'Union européenne. En soulignant en particulier le rôle spécifique de la Commission ainsi que la plasticité du système global, elle ont permis d'explorer plusieurs questions d'une manière plus rigoureuse que par le passé : la mise sur agenda des problèmes sociaux au niveau européen, la spécificité des ressources et des stratégies menées par les acteurs et leurs 
conséquences institutionnelles. Si les approches " interdépendantistes " ont contribué à rendre la " boîte " moins " noire ", peut-on dire pour autant qu'elles ont permis de passer d'une description détaillée de l'intégration européenne à l'analyse des rapports qui lui sont sous-jacents? A-t-on du reste choisi la bonne " boîte " ? Un sentiment de doute face à une approche qui a pourtant inspiré une bonne part de nos propres travaux $^{52}$ nous amène à l'entrevoir de manière critique. Nous examinerons plus particulièrement trois risques: la sectorisation des problématiques, la dérive vers le fonctionnalisme et l'occultation des phénomènes de représentation et de symbolique politiques. Agenda européen ou sectorisation des problématiques? Les recherches consacrées à la mise sur agenda des politiques publiques au niveau européen soulignent le passage d'une situation où globalement les acteurs nationaux " définissaient quels étaient les problèmes à traiter et sous quelle forme ce traitement allait avoir lieu " à une situation où " dans un nombre de domaines toujours plus grand, ce processus de définition des problèmes devant faire l'objet d'une intervention publique est transféré, pour une part plus ou moins grande, au niveau européen "53. Les travaux menés dans cette perspective soulignent deux caractéristiques. D'une part, la mise sur agenda au niveau européen est encore moins linéaire ou a contrario plus circulaire que ce que l'on observe au niveau national ou local. Dans la tradition américaine de l'analyse des politiques publiques ${ }^{54}$, un nombre important des travaux empiriques (sur la concurrence, la recherche et le développement technologique, les fonds structurels...) ont ainsi souligné combien la définition des problèmes à traiter trouvait son origine bien en amont des décisions adoptées par le Conseil de l'Union ${ }^{55}$ et s'enchevêtrait avec les normes communautaires lors de leur mise en œuvre ${ }^{56}$. D'autre part, les études portant sur la mise sur agenda au niveau européen soulignent que ce déplacement s'accompagne d'une transformation des acteurs impliqués. Les acteurs généralistes (hommes politiques, hauts fonctionnaires interministériels) perdent prise sur la formulation des problèmes au profit des " experts " spécialisés. Ces derniers sont essentiellement de trois types. Ce sont les représentants des ministères nationaux siégeant dans la pléthore de comités qui préparent les réunions de la Commission et du Conseil de l'Union. Ce sont les fonctionnaires de la Commission qui utilisent de façon " entrepreneuriale " leur droit d'initiative pour ouvrir de nouveaux " chantiers " communautaires ${ }^{57}$. Enfin, ce sont les représentants des groupes d'intérêt qui ont la possibilité de peser sur des processus de formulation de politiques publiques plus ouverts qu'au niveau national ${ }^{58}$. S'il ne fait aucun doute que les recherches effectuées sur ces questions ont enrichi notre connaissance sur un certain nombre de processus à l'œuvre, permettent-elles pour autant de conclure, comme le fait Pierre Muller ${ }^{59}$, à l'émergence d'un " agenda politique européen " ? Les contributions de Luc Rouban et de Mireille Pongy dans cette revue tendent plutôt à démontrer la difficulté d'identifier un agenda, de le qualifier d'européen, voire d'accorder aux priorités sectorielles le statut de politique publique. Se trouve ainsi posé le risque d'une sectorisation de la recherche sur les politiques publiques européennes. Celle-ci découle moins des limites qu'impose la circonscription des terrains de recherche (on n'évite jamais les limites d'un travail empirique) que d'une difficulté à problématiser. Cette difficulté est particulièrement frappante à la lecture de nombreuses études de cas que publie une revue comme le Journal of European Public Policy. Pour revenir aux propos de J.-C. Thoenig cités plus haut, il existe un danger de " routinisation " des études centrées sur les politiques publiques communautaires, tant sur le plan des méthodes d'enquête employées (essentiellement des entretiens avec les fonctionnaires et les groupes 
d'intérêts), que de la présentation des résultats. Cette dernière ressemble plus souvent à des analyses de " super-experts " qu' à des " synthèses " qui seraient la marque d'une approche plus scientifique ${ }^{60}$. Exprimé plus brutalement, la sectorisation de la recherche sur l'espace européen d'action politique court le risque de déboucher davantage sur des méthodologies propres au management public qu'à la science politique ${ }^{61}$. Ce dernier point a des conséquences théoriques directes. En collant aux propos des acteurs, les études sectorielles contribuent peu à une réflexion plus fondamentale sur la dynamique et le sens de l'intégration européenne. Mais comment aller vers le " haut " en partant $\mathrm{du}$ " bas " sans s'arrêter en cours de route faute de fil conducteur? En privilégiant l'importance historique des rapports entre politiques sectorielles, système politique et ordre social, Bruno Jobert offre une piste intéressante lorsqu'il écrit que ce qui est en cause aujourd'hui en Europe " c'est moins une évolution interne du régime de citoyenneté que son articulation sur un modèle de développement et, plus précisément, sur une architecture spécifique de compromis institutionnalisés "62. A partir des propos de B. Jobert, il est ainsi pertinent de se demander si l'Union européenne s'achemine inévitablement vers un Etat où le politique interviendrait aussi lourdement dans la structuration de la société qu'il ne le fit dans les Etats Providence pendant la période 1945-1980. Une autre thèse intéressante conduit au contraire à se demander si le " caractère hybride de l'entité européenne " ne correspondrait pas à la nature " même de la réalité socio-politique, nationale internationale et transnationale dans laquelle nous entrons, et qui serait caractérisée, par l'interpénétration des unités et des domaines, la multiplicité des allégeances et des compétences, et leur mobilité ou leur fluidité ? "63. Sans que leurs utilisateurs adhèrent nécessairement à cette deuxième orientation, les analystes de l'Union européenne ayant recours aux réseaux, aux politiques régulatrices et à la gouvernance s'efforcent tous de saisir cette fluidité. Réseaux, politiques de régulation et risques du fonctionnalisme A partir des contours de cet espace européen d'action publique, la littérature s'inscrivant dans cette perspective met en avant deux caractéristiques qui marquent à la fois les processus d'entérinement de la règle au niveau communautaire et la transformation des normes et des procédures en actes dans les Etats membres. La première caractéristique est l'absence d'un axe vertical de pouvoir au sein de l'Union européenne due à la prédominance de formes d'action publique fortement interdépendantes et/ou inter-institutionnelles. Souvent saisies à l'aide du concept de réseau d'action publique (policy network) ${ }^{64}$, ces formes se caractérisent par des configurations d'acteurs qui tendent non pas à se conformer à un modèle unique mais à se différencier par l'émergence progressive de règles du jeu internes à chaque domaine. Cette différentiation alimente et s'alimente de modes opératoires de la décision reposant sur une " négociation continue "65. La seconde caractéristique est la faible dimension redistributive des politiques communautaires. Des auteurs comme Giandomenico Majone ont montré que, mis à part la politique agricole commune et les fonds structurels, la plupart des instruments de l'Union européenne correspondent à des politiques de régulation (au sens de la typologie de $\mathrm{T}$. Lowi) ${ }^{66}$, que ce soit dans les domaines de la concurrence ou de la protection de l'environnement. A l'instar du droit post-moderne dont parle Pierre Lascoumes, les politiques régulatrices sont davantage centrées sur " l'élaboration de principes de gestion et l'organisation de procédures d'intervention que sur la définition de normes substantielles " ${ }^{67}$. Dans le cas précis de l'Union européenne, elles comportent en outre deux spécificités. D'une part, elles permettent à la Commission européenne d'élargir son champ de compétences sans accroissement de budget ${ }^{68}$. D'autre part, elles 
s'emboîtent à un système institutionnel qui privilégie la négociation. Majone observe ainsi que " la négociation apparaît comme un trait essentiel de la mise en œuvre de la réglementation. Quel qu'en soit le contenu, la réglementation n'est pas simplement un processus où les autorités commandent et l'acteur réglementé obéit. Une forme de marché apparaît où les bureaucraties et les acteurs soumis à la réglementation négocient sur les obligations précises qu'elle comporte " ${ }^{69}$. Si les réseaux d'action publique et les politiques régulatrices constituent des notions intermédiaires très utiles pour décrire un espace politique polycentrique en action, leur utilisation pourrait quelquefois être " poussée " davantage. Pour ce qui est des réseaux, les analyses portant sur les politiques publiques communautaires (en particulier celles publiées en langue anglaise) négligent le plus souvent deux éléments essentiels : la dimension cognitive et la différence entre mobilisation et pouvoir politique. Sur le premier point, l'analyse reste souvent transactionnelle, c'est à dire qu'elle privilégie les contacts observables entre acteurs institutionnels mais elle tend à négliger les représentations sociales qui portent ces mêmes acteurs. Cette insuffisante attention accordée aux représentations sociales et aux idées permet d'aboutir à la deuxième faille des analyses de l'Union européenne s'appuyant sur la notion de réseau d'action publique: une tendance à traiter la mobilisation des acteurs comme une évidence de leur puissance au niveau communautaire. On se limite alors à présenter l'Union européenne comme le paradis $\mathrm{du}$ lobbying et des lobbyistes ${ }^{70}$ alors que l'accès et l'insertion des acteurs dans les réseaux se construisent dans la durée et exigent une analyse diachronique des rapports d'interdépendance ${ }^{71}$. Cette critique nous ramène au risque qu'encourt la recherche sur les politiques publiques en " collant " trop directement aux paroles des acteurs. Les travaux de S. Mazey et J. Richardson sur le lobbying communautaire constituent un exemple d'une déconstruction insuffisante des paroles des interviewés ainsi que des représentations sociales qu'elles révèlent. Quant à l'analyse des politiques de régulation, elle est intéressante à partir du moment où elle dépasse le constat que ces politiques sont faiblement " budgétivores " et favorables à l'expansion bureaucratique de la Commission pour aller vers une interrogation sur la représentation politique. A partir des travaux de G. Majone, Pierre Muller souligne ainsi l'intérêt d'analyser les tensions sociales naissant du fait que ces politiques régulatrices " ne mettent pas aux prises des groupes ou classes sociales qui s'affronteraient pour modifier à leur profit le partage des ressources, mais des groupes et acteurs qui s'affrontent pour la résolution d'un problème "72. Cette idée d'un débat public concentré sur la " résolution de problèmes " conduit effectivement à interroger la remise en cause par l'Union européenne de " la fonction tribunitienne de la représentation politique, non pas du point de vue du nombre d'électeurs concernés (au contraire!) mais en capacité à articuler et à intégrer le discours protestataire "73. De plus en plus utilisée dans la recherche contemporaine sur les niveaux national ${ }^{74}$ et local ${ }^{75}$, la notion de gouvernance constitue une dernière caractéristique des approches en termes d'espace européen d'action politique. Les partisans de ce terme ${ }^{76} \mathrm{y}$ trouvent généralement quatre avantages pour la construction de leur objet de recherche. Il permet de dépasser l'analyse de l'Union européenne à partir du simple dispositif institutionnel formel. Dans un système politique polycentrique, voire " a-centrique "77, comme celui de l'Union européenne, la gouvernance permet d'identifier les acteurs à travers la généalogie de coalitions qui comprennent non seulement les groupes d'intérêt mais aussi les entreprises, les individus, etc. La gouvernance souligne l'importance de revaloriser les études sur la mise en œuvre dans la mesure où, comme le souligne 
Mireille Pongy, celle-ci " n'est pas une donnée, elle n'est pas une position qui se prend. Elle se construit à travers les interactions des acteurs et est donc à maintenir en permanence "78. Les études sur d'autres secteurs, comme la politique de la cohésion économique et sociale, confirment largement cette hypothèse ${ }^{79}$. La gouvernance a donné naissance à une variante qui tente d'aborder les différentes " échelles " de pouvoir au sein de l'Union européenne : il s'agit du " multi-level governance " dont la traduction française la plus appropriée semble être la " gouvernance à niveaux multiples ". Introduite par des chercheurs comme Gary Marks ou Liesbet Hooghe ${ }^{80}$, cette notion tente de saisir une " configuration en évolution " au sein de laquelle les institutions de l'Union européenne consolident leurs pouvoirs, les Etats perdent les leurs, et un troisième niveau - la région - émerge comme un acteur à part entière. Nous reviendrons plus loin sur le caractère trop systématique de cette thèse au plan empirique et surtout théorique ${ }^{81}$. La question de fond qu'elle tente d'aborder, à savoir la détermination des articulations entre les différents niveaux institutionnels qui composent l'Union européenne, n'en demeure pas moins importante. La gouvernance est présentée enfin comme un moyen de reformuler le rapport entre intégration européenne et théorie démocratique. Plutôt que d'avaliser les regrets des acteurs et des médias sur les faiblesses du Parlement Européen ou sur une Commission " technocratique ", les partisans de la gouvernance tentent de considérer le défi posé par " la pluralité grandissante des intérêts " qui engendre " la dispersion croissante du pouvoir dans de multiples arènes et parmi de multiples acteurs (ce qui curieusement, mais logiquement, rend ce pouvoir dispersé plus oppressif pour chacun puisqu'il n'est imputable à personne) "182. Les analyses utilisant la notion de gouvernance ont effectivement permis de décrire certaines tendances significatives du politique au sein de l'Union européenne, apportant ainsi des éléments majeurs au débat. Il n'empêche que ces analyses souffrent souvent aussi de trois failles: un amalgame entre convergence et ressemblance des modes d'action politique, une interrogation insuffisante sur la représentation politique, et une occultation de la question du " qui domine? " la gouvernance. Avancée comme une théorie à moyenne portée par des chercheurs comme S. Andersen et K. Eliassen ${ }^{83}$, " l'européanisation " ou " l'européification " de l'action politique est vue comme un élément moteur d'une " convergence " des systèmes politiques nationaux vers un nouveau modèle politique européen. Cette hypothèse de la convergence mérite d'être traitée avec prudence tant au plan empirique que théorique. En orientant de nombreuses études de cas sur les politiques communautaires, elle débouche en effet trop souvent sur la transformation de la convergence en une fin en soi, et d'autre part, sur une confusion entre convergence et ressemblance des modes d'action publique. Elle conduit alors à oublier que la mise en œuvre des politiques communautaires ne peut pas être étudiée en dehors de la prise en compte de modèles (patterns) de gouvernance qui sont spécifiques à des territoires (notamment au sein des Etats-Nations). Ces modèles ou, pour reprendre les termes de Weber, ces " ordres légitimes de pouvoir ", sont constitués non seulement de configurations particulières d'institutions et de procédures formelles mais aussi d'institutions sociales, comme le service public ou la sécurité sociale, qui structurent le sens accordé à l'action publique. Presque toutes les approches de l'Union européenne en termes de gouvernance négligent une deuxième question essentielle: celle de la représentation politique, qu'un auteur comme J. Leca met lui bien en relief au plan national. En lisant ces travaux, on est en effet frappé par la faible prise en compte des hommes politiques impliqués dans les politiques communautaires et par 
une conceptualisation rigide de l'articulation entre administration et politique. La première omission relève de l'attention généralement trop grande accordée aux experts dans les études partant des réseaux d'action publique ${ }^{84}$. Elle trouve en outre ses racines dans une conception du politique qui minimise l'importance du discours, des prises de position, des mises en scène, en quelque sorte de l'évocation telle qu'elle a pu être formulée par l'anthropologue Marc Abélès ${ }^{85}$. De façon étonnante, cette vision " techniciste " du politique est présente y compris dans de nombreuses études portant sur le rôle du Parlement européen au sein du système communautaire. Celles-ci se bornent en effet à étudier les stratégies adoptées par les groupes politiques par rapport aux procédures d'" avis conforme " ou de " co-décision " sans s'interroger sur les conditions d'émergence d'une rhétorique politique transnationale ou sur la manière dont les parlementaires européens présentent ou représentent l'Europe dans leurs pays respectifs ${ }^{86}$. Les quelques études approfondies sur le rôle des commissaires ou sur la présidence du Conseil européen évacuent tout autant le problème de la représentation ${ }^{87}$. Cette conception " désincarnée " du politique est souvent amplifiée lorsque l'attention se porte sur la morphologie du système politique global. Non seulement les acteurs mais aussi les institutions dans lesquelles ils siègent (notamment le Conseil des ministres et le Conseil européen) se trouvent relégués au rang de simples contraintes exogènes à la dynamique des réseaux. Le schéma d'analyse du processus décisionnel de l'Union européenne proposé par John Peterson ${ }^{88}$ en est une illustration. L'auteur postule en effet que l'on peut distinguer trois types de décisions au niveau communautaire. Les history-making decisions qui modifient les procédures législatives de l'Union, l'autorité des institutions et délimitent les domaines d'intervention; c'est un niveau dominé par le Conseil européen et la Cour de justice des Communautés. Les policy setting decisions qui sélectionnent les formes d'action à entreprendre ; c'est un niveau dominé par le Conseil des ministres et par les gouvernements nationaux. Les policy shaping decisions qui abordent la question de " comment atteindre les objectifs que le Conseil a arrêté ? " ; c'est un niveau dominé par la Commission et par les comités d'experts nationaux. Outre la linéarité discutable de ce schéma, on y trouve une distinction confuse entre rationalités administrative et politique. En examinant la nature de la Commission, l'auteur a d'ailleurs du mal à s'y retrouver lui-même dans ses propres catégories. Comme nous l'avons vu plus haut, une tentative de contournement de cette confusion a été, depuis trois ou quatre ans, le recours à la notion de multi-level governance. Mais la plupart des études publiées sous ce label se cantonnent hélas à une approche institutionnaliste qui minimise les rapports entre société et politique et favorise une vision intergouvernementaliste de l'intégration européenne. A cet égard, il n'est pas anodin que les études sur le multi-level governance s'intéressent beaucoup plus aux niveaux (" levels ") institutionnels qu'aux conflits et aux concurrences interterritoriales. L'étude de l'Union européenne comme système de gouvernance nous informe enfin très peu sur la constitution de hiérarchies d'acteurs et d'intérêts. L'occultation de la question de l'autorité découle de l'héritage pluraliste qui inspire la plupart des études s'y rattachant. Partant de l'intéressant postulat de recherche selon lequel les hiérarchies ne se décréteraient plus au sein de l'Union européenne, elles aboutissent souvent à la conclusion plus contestable que les hiérarchies n'y existeraient plus ${ }^{89}$. Or tout l'intérêt (mais aussi toute la difficulté) d'analyser l'Union européenne consiste précisément à identifier des hiérarchies qui ne sont pas " données " à l'avance mais qui se composent, se décomposent et se recomposent dans la négociation. CONCLUSION Il ne fait aucun doute que la densité descriptive des études de science 
politique sur l'Union européenne commence à devenir impressionnante. A quelques exceptions près, ces études ne vont toutefois pas assez loin dans la conceptualisation de questions aussi fondamentales que la dimension cognitive et symbolique du politique en Europe, les modes de représentation politique ou encore le rapport entre espaces de négociation catégoriels et configurations sociales. L'intergouvernementalisme libéral comme les approches en termes d'espace européen d'action politique (malgré leur relative nouveauté) semblent ainsi peu inclines à revisiter vraiment les recherches empiriques qu'elles alimentent. Comme nous nous efforcerons de le montrer dans l'article de conclusion de ce numéro, c'est donc d'une relance théorique dont a besoin la recherche politologique sur l'Union européenne ou encore d'un effort de dépassement de questionnements devenus trop confortables pour être suffisants.

\section{NOTES}

1. Nous tenons à remercier pour leurs commentaires N. Jabko, A.-M. Le Gloannec et M. Pongy.

2. Respectivement Chargés de recherche au CERI-FNSP et au CERVL-FNSP.

3. G. Tordjman, " Le silence de la science politique ", Libération, 2-3 novembre 1996.

4. Thomas Risse-Kappen, " Exploring the nature of the beast : International relations theory and comparative politics analysis meet the European Union ", Journal of Common Market Studies, 34/1, march 1996, pp. 53-80.

5. E. Haas, The obsolescence of regional integration theory, Berkeley, Center for International Studies, 1975.

6. J. Caporaso et J. Keeler, " The European Union and regional integration theory " in Carolyn Rhodes and Sonia Mazey (eds), The state of the European Union, vol. 3, Boulder, Lynne Rienner, 1995, p.27 et 42.

7. A. Sbragia (ed.), Euro-politics : institutions and policymaking in the " new " European Community, Washington, Brookings Institution, 1992 ; et aussi Simon Hix, " The study of the European Community : the challenge to comparative politics ", West European Politics, 17/1, January 1994, pp. 1-30.

8. A-M. Burley, W. Mattli, " Europe before the court : a political theory of legal integration ", International Organization, n 47, 1993 ; et aussi K. Alter, S. MeunierAitsahalia, "Judicial politics in the European Community : European integration and the pathbreaking Cassis de Dijon decision ", Comparative Political Studies, $n^{\circ} 26$, January 1994.

9. En France, les années quatre-vingt se caractérisent aussi par une forte empreinte du paradigme transnational sur les travaux théoriques de relations internationales (il est vrai pas si nombreux que cela). Les recherches menées par Bertrand Badie et MarieClaude Smouts ont en particulier marqué le débat intellectuel (voir Le retournement du monde, Paris, Presses de Science Po, 1992) ainsi qu'une génération de doctorants formés à l'Institut d'Etudes Politiques de Paris.

10. S. Hoffmann, " Europe's identity crisis : Between the past and America " (1964), reproduit in Essays on Europe 1964-1994, Boulder, Westview Press, 1995, pp. 9-50 ; et 
aussi " Obstinate or obsolete? The fate of the nation-state and the case of Western Europe ", Daedalus, Summer 1966, pp. 862-915.

11. R. Keohane et S. Hoffmann (eds), The new European Community : decision-making and institutional change, Boulder, Westview, 1991.

12. G. Garrett, "International cooperation and institutional choice : the European Community's internal market " in John G. Ruggie (ed.), Multilateralism matter. The theory and praxis of an international system, New York, Columbia University Press, 1993.

13. Alors qu'une grande majorité des politistes anglophones (et tout particulièrement britanniques) s'intéressant à l'intégration européenne limitent de plus en plus leurs lectures aux travaux publiés dans leur seule langue, A. Moravcsik utilise au contraire un matériel empirique constitué de références en langue anglaise, allemande et française. Cette qualité est devenue suffisamment rare pour être soulignée.

14. Pour une critique des travaux de Moravcsik, voir D. Wincott, " Institutional interaction and European integration : towards an everyday critique of liberal intergovernmentalism ", Journal of Common Market Studies, 33/4, December 1995, pp. 597-609.

15. A. Moravcsik, " Preferences and power in the European Community : a liberal intergovernmentalist approach ", Journal of Common Market Studies, 31/4, December 1993, pp. 473-524.

16. A. Moravcsik, " Negotiating the Single European Act : national interests and conventional statecraft in the European Community ", International Organization, Winter 1991, pp. 19-56.

17. E. Cohen, La tentation hexagonale, Paris, Fayard, 1996.

18. E. Cohen, op. cit., p. 317.

19. J. J. Anderson, " The state of the (European) Union : from the single market to Maastricht, from singular events to general theories ", World Politics, April 1995, pp. 441-465.

20. A. Moravcsik, op.cit., 1991, pp. 48-49 ; souligné par nous.

21. H. Wallace, " Making multilateralism work " in William Wallace (ed.), The dynamics of European integration, London, Pinter, 1990.

22. A. Moravcsik, Why the European Community strengthens the state : domestic politics and international cooperation, Harvard Center for European Studies, working paper $n^{\circ} 52,1994$.

23. A. Moravcsik, op. cit., 1993, p. 507.

24. Chr. Lequesne, " La Commission européenne entre autonomie et dépendance ", Revue Française de Science Politique, 46/3, juin 1996, pp. 389-408; et aussi Wayne Sandholz, "Membership matters : limits of the functional approach to the European institutions ", Journal of Common Market Studies, 34/3, September 1996, pp. 403- 429. 25. L. Cram, " The European Commission as a multi-organization : social policy and IT policy in the EU ", Journal of European Public Policy,1/1994, pp. 195-218.

26. S. Bulmer, " Domestic Politics and European Community policy-making ", 21/4, June 1983, pp. 349-363.

27. R. Putnam, " Diplomacy and domestic politics : the logic of two-level games ", International Organization, Summer 1988, pp. 427-461.

28. A. Moravcsik, op.cit., 1994 ; voir aussi A. Milward, The European rescue of the nation-state, Berkeley, University of California Press, 1992.

29. Chr. Lequesne, op.cit., 1993. 
30. A. Moravcsik, op. cit., 1993, p. 483 ; pour une critique, voir Gary Marks, Liesbet Hooghe et Kermit Blank, " European integration from the 1980s ", Journal of Common Market Studies, 34/3, September 1996, pp. 341-378.

31. P. Muller, "Introduction. Un espace européen des politiques publiques " in Y. Mény, P. Muller et J.-L. Quermonne (dir.), Politiques publiques en Europe, Paris, L'Harmattan, 1995.

32. A. Moravcsik, op. cit., 1993, p. 45.

33. W. Sandholtz, J. Zysman, " Recasting the European bargain ", World Politics, October 1989, pp. 95-128.

34. Cf. infra.

35. Pascal Vennesson, critique bibliographique in Revue Française de Science Politique, 45/5, octobre 1995, pp. 857-866.

36. A. Moravcsik, op. cit., 1993, p. 477.

37. J. Snyder, " Richness, rigor and relevance in the study of Soviet foreign policy ", in Sean Lynn-Jones, S. Miller et Stephen Van Evera (eds), Soviet military policy, Cambridge, MIT, 1989, pp. 3-22.

38. S. Andersen, K. Eliassen (eds), Making policy in Europe. The Europification of national policy-making, London, Sage, 1993.

39. P. Muller, op. cit., 1995.

40. Jean-Claude Thoenig signale ainsi trois dangers pour l'analyse des politiques publiques telle qu'elle est pratiquée en France : la routinisation des problématiques et des méthodes, la vulgarisation d'une approche scientifique en prescriptions de management public et un complexe d'infériorité par rapport à d'autres branches de la sociologie et de la science politique, in " La quête du deuxième souffle ", Revue française de science politique, 46 (1), février 1996. Patrice Duran va plus loin en évoquant trois autres questions dans son article " L'analyse des politiques publiques en perspective, de la crise du politique à sa reconstruction ", Revue française de science politique, 46 (1), février 1996. A une époque de " reconstruction du politique ", il est tout d'abord temps pour lui de dépasser un questionnement en terme d'efficacité de l'action publique pour s'interroger plus systématiquement sur la " publicness " (p. 110) et la nature même de la responsabilité politique (pp. 117-118). Deuxièmement, il ne suffit plus de rester au niveau des changements de dispositifs institutionnels et de procédures, une attention plus grande devant être portée aux résultats du point de vue des publics concernés et de leur imbrication avec le changement social (p. 111). Enfin, une reconnexion entre analyse des politiques publiques et analyse des institutions doit être opérée dans la mesure où " tout n'est pas processus " (p. 115).

41. C. Webb, "Theoretical perspectives and problems " in W. Wallace, H. Wallace \& C. Webb (eds), Policy-making in the European Community, London, J. Wiley, 1983.

42. J. Jamar et W. Wessels (dir.), L'administration communautaire à l'heure des choix, Bruges, De Tempel, 1985.

43. H. Siedentopf, J. Ziller (dir.), L'Europe des administrations ? : la mise en oeuvre de la législation communautaire dans les Etats membres, Londres, Sage, 2 vol. 1988.

44. Wolfgang Wessels, " Staat und (westeuropäische) Integration. Die Fusionsthese " in Michael Kreile (hrsg.), Die Integration Europas, Opladen, Westdeutscher Verlag, 1992.

45. F. Scharpf, " The joint-decision trap : lessons from German federalism and European integration " in Law and state, volume 36, 1987.

46. M. Croisat et Jean-Louis Quermonne, L'Europe et le fédéralisme : contribution à l'émergence d'un fédéralisme intergouvernemental, Paris, Montchrestien, 1996. 
47. R. Keohane, " The demand for international regimes ", International Organization, XXXVI, (2), 1982 ; S. Krasner (ed.), International regimes, Ithaca, Cornell University Press, 1983.

48. $P$. Soldatos, Le système institutionnel et politique des Communautés européennes dans un monde en mutation : théorie et pratique, Bruxelles, Bruylant, 1989 ; E. Kirchner, Decision-making in the European Community. The Council presidency and European integration, Manchester, Manchester University Press, 1992.

49. M. Sharpe, in J. Lodge (ed.), The EC and the challenge of the future, London, Pinter, 1990.

50. E. Cohen, " A la recherche de l'adversaire et politique économique : nationalisation, politique industrielle et Acte Unique Européen ", Revue francaise de science politique, vol. 43 (5), 1993.

51. H. Wallace, " Negotiations and coalition formation in the European Community ", Government and Opposition, 20 (4), autumn 1985.

52. Chr. Lequesne, Paris-Bruxellles. Comment se fait la politique européenne de la France, Paris, Presses de Science Po, 1993 ; A. Smith, L'Europe politique au miroir du local, Paris, L'Harmattan, 1996.

53. P. Muller, op cit., 1995, p. 14.

54. J. Pressman et A. Wildavsky, Implementation, Los Angeles, University of California Press, 1973.

55. G. Peters, " Agenda-setting in the European-Community ", Journal of European Public Policy, vol. 1 (1), 1994.

56. H. Dumez et A. Jeunemaître, La concurrence en Europe. De nouvelles règles du jeu pour les entreprises, Paris, Seuil, 1991 ; L. Jourdain, Recherche scientifique et construction européenne, Paris, L'Harmattan, 1995.

57. L. Cram, " The European Commission as a multi-organisation ", Journal of European public policy, vol. 1 (2), 1994. Voir aussi un numéro spécial de la Revue francaise de science politique sur la Commission européenne paru en juin 1996.

58. S. Mazey et J. Richardson, (eds), Lobbying in the European Community, Oxford, Oxford University Press, 1993.

59. Op. cit., 1995, p.14.

60. N. Elias, Qu'est-ce que la sociologie ?, La Tour d'Aigues, L'Aube, 1991.

61. Bien entendu, les méthodologies de management public posent des questions de même ordre, comme par exemple la " performance institutionnelle ".

62. B. Jobert, " Le retour tâtonnant de l'Etat ", in F. d'Arcy et L. Rouban (dir.), De la Ve République à l'Europe, hommage à Jean-Louis Quermonne, Paris, Presses de Sciences Po, 1996, p. 318.

63. P. Hassner, " Ni sang ni sol ? Crise de l'Europe et dialectique de la territorialité " in B. Badie et M.-C. Smouts (dir.), L'international sans territoire, numéro spécial de Cultures \& Conflits, été 1996, p. 118.

64. J. Peterson, " Decision-making in the European Union : towards a framework for analysis ", Journal of European Public Policy, 2 (1), 1995.

65. M. Abélès, En attente d'Europe, Paris, Hachette, 1996.

66. G. Majone, " CEE : déréglementation ou re-réglementation ? La conduite des politiques publiques depuis l'Acte Unique " in B. Jobert (dir.), Le tournant néo-libéral, Paris, L'Harmattan, 1994.

67. P. Lascoumes, " Normes juridiques et mise en oeuvre des politiques publiques ", L'Année sociologique, 1990, p. 71. 
68. L. Cram, " Calling the tune without paying the piper ? Social policy regulation : the role of the Commission in EC social policy ", Policy and Politics, 21 (2), 1993.

69. G. Majone, op. cit., 1994, p. 255.

70. S. Mazey et J. Richardson (eds), op. cit., 1993.

71. E. Négrier, " Intégration européenne et échanges politiques territorialisés ", Pôle sud, $\mathrm{n}^{\circ} 3,1995$.

72. P. Muller, " Politiques publiques et citoyenneté en Europe ", contribution aux travaux du groupe CERI-CERAT, dactyl., 1995, p. 4.

73. Ibid, p. 5.

74. J. Leca, " La gouvernance de la France sous la Ve République : une perspective de sociologie comparative ", in F. d'Arcy et L. Rouban, (dir.), op.cit..

75. G. Stoker, " Local governance, a conceptual challenge ", communication aux sessions de l'ECPR, Bordeaux, mai 1995 ; P. Le Galès, " Du gouvernement des villes à la gouvernance urbaine ", Revue francaise de science politique, vol. 45 (1), 1995.

76. M.-C. Smouts, " Du bon usage de la gouvernance en relations internationales ", à paraître dans la Revue Internationale des Sciences Sociales.

77. P. Durand et J-C. Thoenig, " L'Etat et la gestion publique territoriale ", Revue francaise de science politique, vol. 46 (4), 1996, p. 580.

78. M. Pongy, manuscrit du texte publié in G. Saez, J-Ph. Leresche et M. Bassand (dir.), Gouvernance métropolitaine et transfrontalière : action publique territoriale, Paris, L'Harmattan, 1997.

79. M. Smyrl, " Does EC regional policy empower the regions ? ", communication à la Conference of Europeanists, Chicago, mars 1996.

80. G. Marks, " Structural policy in the European Community ", in A. Sbragia, (dir.), Europolitics, Washington, Brookings Institute, 1992 ; Liesbet Hooghe (ed.), Cohesion Policy and European integration : building multi-level governance, Oxford, Oxford University Press, 1996.

81. Ch. Jeffrey, " The emergence of multi-level governance in the European Union ", à paraître in Politiques et management public, 1997 ; P. Le Galès et C. Lequesne (dir.), Les paradoxes des régions en Europe, Paris, La Découverte, 1997.

82. J. Leca, op. cit., 1996.

83. Andersen et Eliassen, Op. cit., 1993.

84. Marsh et Rhodes ont pu ainsi noter que " la légitimité des réseaux n'est pas politique. Elle réside dans la prétention d'un acteur à véhiculer une expertise supérieure et/ou de pouvoir fournir un service de manière plus efficace " ; D. Marsh \& R. Rhodes (eds), Policy networks in British government, Oxford, Clarendon Press, 1992, p. 265.

85. M. Abélès, Jours tranquilles en 89 . Ethnologie politique d'un département français, Paris, Odile Jacob, 1989.

86. Une exception étant le livre de Marc Abélès, La vie quotidienne au Parlement européen, Paris, Hachette, 1992.

87. G. Ross, Jacques Delors and European integration, New York, Oxford University Press, 1995 ; E. Kirchner, op. cit., 1992.

88. Peterson, Op. cit., 1995, pp. 72-76.

89. D. Sidjanski, L'avenir fédéraliste de l'Europe, Paris, PUF, 1992 
INDEX

Mots-clés : régionalisme et régionalisation, Relations Internationales, sciences politiques, construction européenne

Index géographique : Bruxelles, Europe, Union européenne 